\title{
Integration of Cell Membranes and Nanotube Transistors
}

XXXX

Vol. 0, No. 0

$\mathbf{A}-\mathbf{E}$

\author{
Keith Bradley, ${ }^{\dagger}$ Alona Davis, ${ }^{\dagger}$ Jean-Christophe P. Gabriel, ${ }^{\dagger}$ and G. Gruner ${ }^{\star}, \dagger, \ddagger$ \\ Nanomix Inc., Emeryville, California 94608, and Department of Physics and \\ Astronomy, University of California, Los Angeles, California 90095
}

Received January 25, 2005; Revised Manuscript Received March 23, 2005

\begin{abstract}
We report the integration of a complex biological system and a nanoelectronic device, demonstrating that both components retain their functionality while interacting with each other. As the biological system, we use the cell membrane of Halobacterium salinarum. As the nanoelectronic device, we use a nanotube network transistor, which incorporates many individual nanotubes in such a way that entire patches of cell membrane are contacted by nanotubes. We demonstrate that the biophysical properties of the membrane are preserved, that the nanoelectronic devices still function as transistors, and that the two systems interact. Further, we use the interaction to study the charge distribution in the biological system, finding that the electric dipole of the membrane protein bacteriorhodopsin is located $2 / 3$ of the way from the extracellular to the cytoplasmic side.
\end{abstract}

Nanobioelectronics, the integration of biological processes and molecules with nanoscale fabricated structures, offers the potential for electronic control and sensing of biological systems. ${ }^{1}$ As a specific example, carbon nanotubes have been suggested for use as prosthetic nervous implants in organs such as eyes and ears. ${ }^{2}$ To achieve this goal requires the parallel preparation ${ }^{3}$ of fully functional biological systems and nanoelectronic systems that are integrated together. One major obstacle is the preservation of functionality in both systems. For example, while biological systems ranging from lipids $^{7}$ to living cells ${ }^{2}$ have been assembled on nanotube substrates, the nanotubes have served only as mechanical supports, without electronic functionality. A second major obstacle is the difference in scale between nanostructures and biological systems. While nanotubes are comparable in size to individual proteins, they are much smaller than cells. Thus, nanotube electronic devices have been used as singlemolecule sensors ${ }^{5 a, 7 a}$ rather than to communicate with complex biological systems. Here, we achieve integration between a functioning nanotube transistor and a cell membrane. We use nanotube networks, a recently developed class of nanotube devices, to bridge the gap in size between nanotechnology and biotechnology. To demonstrate the power of the approach, we use the nanobioelectronic devices to extract information about the charge distribution in the particular membrane used, thereby contributing to the resolution of a long-standing question about charge distributions within that membrane. ${ }^{6}$

* Corresponding author. E-mail: jcgabriel@nanomix.com; ggruner@ ucla.edu

$\dagger$ Nanomix Inc.

$\doteqdot$ UCLA.
The structure of the devices is illustrated in Figure 1. Patches of cell membrane covered a dense network of individual carbon nanotubes (Figure 1A) contacted by metal electrodes, ${ }^{8}$ referred to as a nanotube network field-effect transistor (NTN-FET). This configuration has several significant features. First, the cell membrane is in direct contact with the semiconducting channel of the transistor. This is distinct from previous work, in which cell membranes have contacted the gate electrodes of transistors. ${ }^{9}$ In this configuration, transistors detect the electrical potential across membranes; in contrast, our devices detect local electrostatic charges on the biomolecules. This is possible because the nanotubes are robust, air-stable semiconductors that can be exposed to cell membranes. Second, the use of a large number of nanotubes ensures that entire patches of membrane are in contact with nanotubes. Thus, the size scale of nanotechnology, which enables the semiconductor integration, is interfaced with the larger size scale of biology. Note that the preservation of transistor operation in a device with many nanotubes requires careful control of the growth parameters (as described in ref 8), because metallic nanotubes will otherwise shunt much of the transistor current.

As the cell membrane, we chose purple membrane (PM) from Halobacterium salinarum, ${ }^{10}$ which has been widely studied. PM contains the light-sensitive membrane protein bacteriorhodopsin, which serves as a photochemical proton pump and has been used to fabricate phototransistors. ${ }^{9}$ In addition, rhodopsin has a permanent electric dipole moment, a charge distribution that produces an electric field pointing from the extracellular side of the membrane toward the cytoplasmic side. ${ }^{9}$ These properties make PM an ideal prototype membrane for nanobioelectronic integration. In 

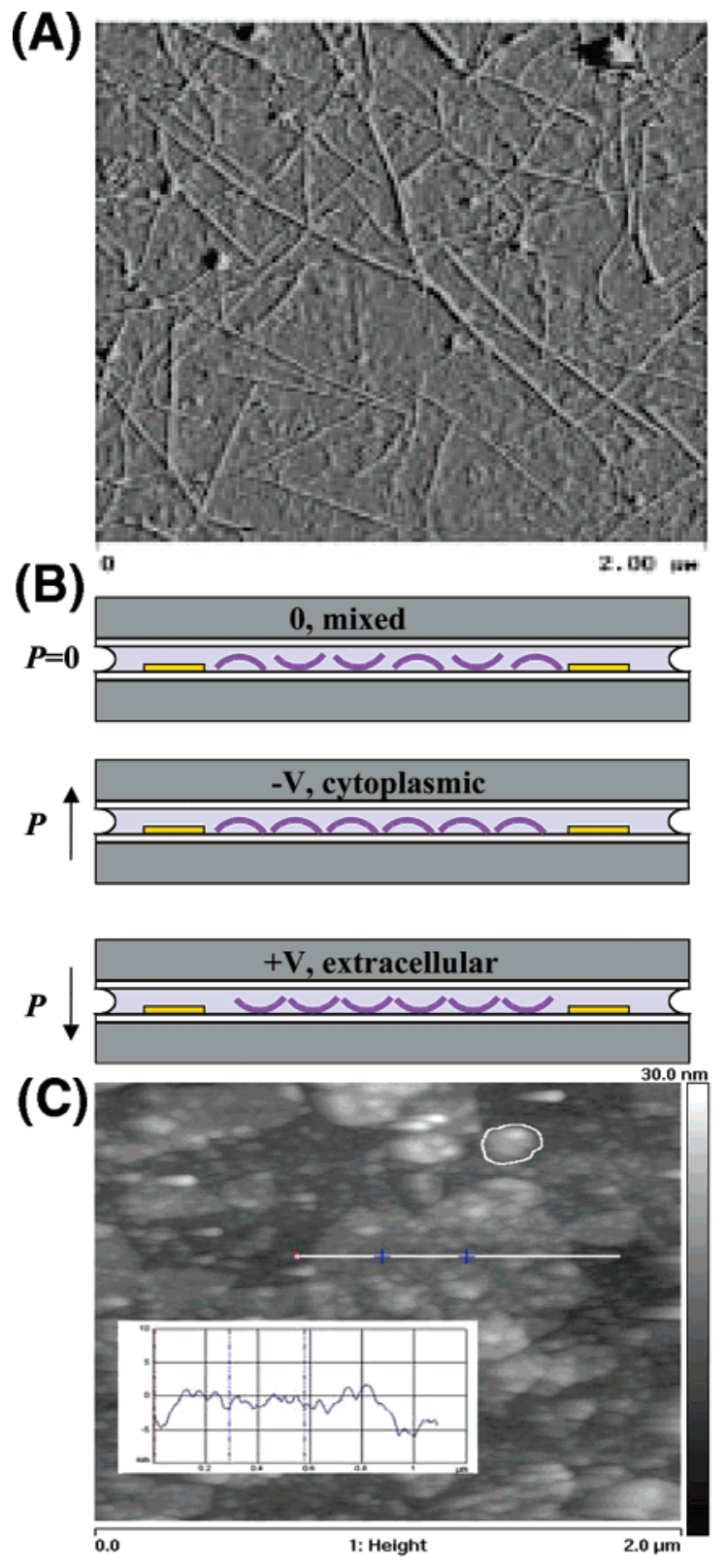

Figure 1. (A) Atomic force microscopy image (amplitude signal) of a nanotube network. The network contains many randomly oriented carbon nanotubes, grown on a substrate (silicon oxide on metallic silicon) by CVD. The nanotubes occur individually, rather than in bundles. The density is adjusted so that the network functions as a transistor, with the gate voltage provided by the buried substrate. (B) Setup for deposition of PM. A silicon chip with a thin oxide coating is placed on top of the sample chip, leaving a few microns of liquid between them. Three deposition conditions were used. At the top, called mixed-orientation, the top chip has zero electrical potential, so that the rhodopsin dipoles point up and down with equal frequency. As a result, the PM contacts the nanotubes with both sides, and the net dipole moment, $P$, is zero. Middle, cytoplasmic orientation, ${ }^{26}$ with $-3 \mathrm{~V}$ on the top chip, so that the net dipole moment is upward. Bottom, extracellular orientation, with $+3 \mathrm{~V}$ on the top chip, so that the net dipole moment is downward. (C) Atomic force microscopy topograph of a completed nanobioelectronic device with mixed-orientation PM coating a nanotube network. PM is visible as irregular patches, one of which is outlined. The white line indicates the contour selected for a line section of the image, shown in the inset. As shown in the inset, the PM patch is, within 30\%, $5 \mathrm{~nm}$ high. particular, we use the dipole as an indicator that the integration preserves the biomaterial while bringing it into contact with the nanoelectronic devices.

PM isolated from Halobacterium salinarum ${ }^{12}$ was deposited on previously fabricated NTN-FETs (see Supporting Information). To observe the effect of the electric dipoles fixed in the PM, devices were prepared in three conditions, as shown in Figure 1B: with the cytoplasmic side of the PM facing the nanotubes, ${ }^{13}$ with the extracellular side facing the nanotubes, ${ }^{13}$ and with a mixture of both orientations. ${ }^{14}$ The films were measured by AFM to be $5 \mathrm{~nm}$ thick, which corresponds to monolayers of PM. ${ }^{9}$ Before and after deposition of PM, the NTN-FET transfer characteristics (conductance versus gate voltage) were measured.

To describe our observations of the devices, we begin with the transfer characteristics before the deposition of PM (Figure 2A). The devices operated as p-type transistors, ${ }^{15}$ conducting well at negative gate voltages and not conducting at positive gate voltages. In the region of zero gate voltage, the devices turned on sharply as the gate voltage was changed; this sharp turn-on, or high transconductance, has been attributed to the high mobility of charge carriers in carbon nanotubes. ${ }^{16}$ The sharp turn-on begins at a specific gate voltage, referred to as a threshold voltage. However, the devices showed significant hysteresis, in that different threshold voltages were measured using left-moving and right-moving sweeps of the gate voltage. The intrinsic threshold voltage is taken to be the average between the leftmoving and right-moving threshold voltages.

Next, we describe the transfer characteristics after the deposition of mixed-orientation PM, in comparison to those before deposition. Figure 2A highlights three main device parameters before and after deposition for a typical device. The changes described here were observed repeatedly in several devices prepared in the same way. First, the hysteresis loops narrowed significantly, as indicated by the arrows. In this case, the width decreased from 3.5 to $0.8 \mathrm{~V}$. Second, the threshold voltage changed by $+1.0 \pm 0.2 \mathrm{~V}$, as indicated by the arrows on the $x$-axis. Finally, the transconductance decreased by about $20 \%$. As discussed below, these changes show that the PM has been successfully integrated with the NTN-FETs.

Finally, we compare the effects of oriented PM deposition. In both orientations (Figures 2B, 2C) the membrane deposition caused a narrowing of the hysteresis loops similar to that caused by the mixed-orientation deposition. For the cytoplasmic orientation, the hysteresis width decreased from 1.2 to $0.8 \mathrm{~V}$; for the extracellular orientation, the hysteresis width decreased from 1.3 to $0.9 \mathrm{~V}$. At the same time, the threshold voltages shifted, in opposite directions according to the orientation of the membrane. For the cytoplasmic orientation (Figure 3A) the threshold voltage shifted by +2.2 $\pm 0.2 \mathrm{~V}$; for the extracellular orientation (Figure 3B) the shift was $-0.4 \pm 0.2 \mathrm{~V}$. Finally, the transconductance did not change, although the maximum conductance changed in accordance with the shifts in the threshold voltage.

We will analyze these observations in the light of what is already known about nanotube transistors. First, we discuss the transconductance. This quantity is associated with the 

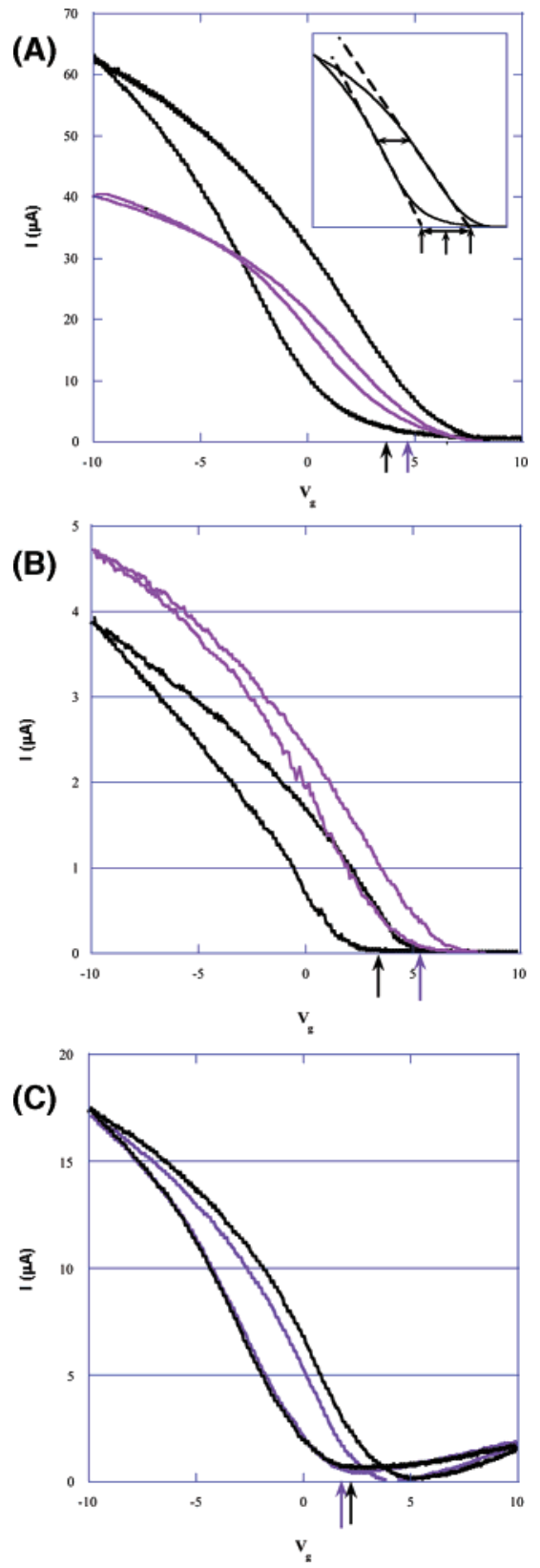

Figure 2. (A) Transfer characteristics (current versus gate voltage) (bias voltage $=100 \mathrm{mV}$ ) for a device before (black) and after (purple) the deposition of cell membrane. Each transfer characteristic has two curves, from the right-moving sweep of gate voltage and the left-moving. The intrinsic threshold voltage, indicated by black and purple arrows respectively, is the average between the two sweeps. (inset) Schematic transfer characteristic, illustrating the calculation of the device parameters. The width of the hysteresis is indicated by the pairs of horizontal arrows, drawn at a conductance of $50 \%$ of the maximum. The transconductance for the right- and left-moving sweeps is shown by dashed lines. Each transconductance is extrapolated back to zero current, where its intersection with the $x$-axis is the right-moving or left-moving threshold voltage. These two threshold voltages are indicated by arrows on the axis. The midpoint between them is the intrinsic threshold voltage, indicated by a third arrow. (B) Transfer characteristics (bias voltage $=100 \mathrm{mV}$ ) before (black) and after (purple) the deposition of membrane oriented with the cytoplasmic side contacting the nanotubes. (C) Transfer characteristics (bias voltage $=100 \mathrm{mV}$ ) before (black) and after (purple) the deposition of membrane oriented with the extracellular side contacting the nanotubes. capacitance between the nanotube network, which forms the channel of the NTN-FET, and the gate; and with the mobility of carriers within the nanotube network. ${ }^{16}$ The capacitance is unlikely to change as a result of membrane deposition, and this notion is confirmed by the fact that the transconductance is not changed by oriented membrane deposition. In the case of mixed-oriented membrane deposition, the alternation of positive and negative electric dipoles on a length scale of about $500 \mathrm{~nm}$ (the diameter of a typical patch of PM) should act as a significant random scattering potential, which decreases the carrier mobility in the network. ${ }^{18}$ Thus, the decrease in transconductance in Figure $2 \mathrm{~A}$ only is a direct result of the mixture of orientations.

Second, the hysteresis decreased dramatically in all cases as a result of the biological coating. The hysteresis is known to result from adsorbed water on the substrate; ${ }^{17}$ in addition, coatings that displace water from the nanotubes reduce the hysteresis. ${ }^{17}$ Consequently, we expect a decrease in hysteresis here as well, presuming that the PM remains intact as a layer contacting the nanotubes. Moreover, the width of the remaining hysteresis is similar for all three conditions, which suggests that the amount of PM coverage is similar. This conclusion was confirmed in randomly selected spots that were imaged by AFM.

Third, the shift of the threshold voltage in the devices results from the electrostatic field associated with the bacteriorhodopsin electric dipole. This field induces charge in the nanotubes, thus shifting the Fermi level. ${ }^{19}$ The position of the Fermi level is measured by the threshold voltage, and an extensive literature has established the relationship between the threshold voltage in various device configurations and the quantity of charge induced in the nanotubes. ${ }^{20-22}$ In our case, with a typical nanotube diameter of $2 \mathrm{~nm}$, every $1 \mu \mathrm{m}$ of nanotube length has a capacitance to the gate, $C_{\mathrm{bg}}$, of $15 \mathrm{aF}^{20}$ The induced charge, $\Delta Q$, is given by $\Delta Q=$ $C_{\mathrm{bg}} \Delta V$, where $\Delta V$ is the threshold shift. Thus, the $+1.1 \mathrm{~V}$ shift caused by mixed-orientation PM deposition corresponds to an induced charge of $16 \mathrm{aC} / \mu \mathrm{m}$ of nanotube length.

Thus, by studying these three device parameters, we conclude the nanobioelectronics integration is successful. First, the NTN-FET transistor functionality is preserved. Second, the PM remains intact as a layer, and the bacteriorhodopsin membrane proteins retain their electric dipoles. Third, the deposited PM has been demonstrated to contact the NTN-FETs directly and to interact with their electrical properties.

Next, we will examine the interaction more closely. In particular, we observe a significant asymmetry between cytoplasmic and extracellular orientations. This asymmetry is reflected in the large amount of charge induced in mixedorientation devices, since without an asymmetry the charge induced by equal amounts of cytoplasmic- and extracellularoriented PM should cancel. ${ }^{23}$ Such an asymmetry is known to exist, in that the dipole is closer to one side of the PM than the other. ${ }^{24}$ Here we are able to observe this asymmetry directly because of the device configuration in which the PM contacts the nanotubes directly.

Furthermore, we will now quantify the asymmetry, by modeling the electrostatic effect of the bacteriorhodopsin 
(A)

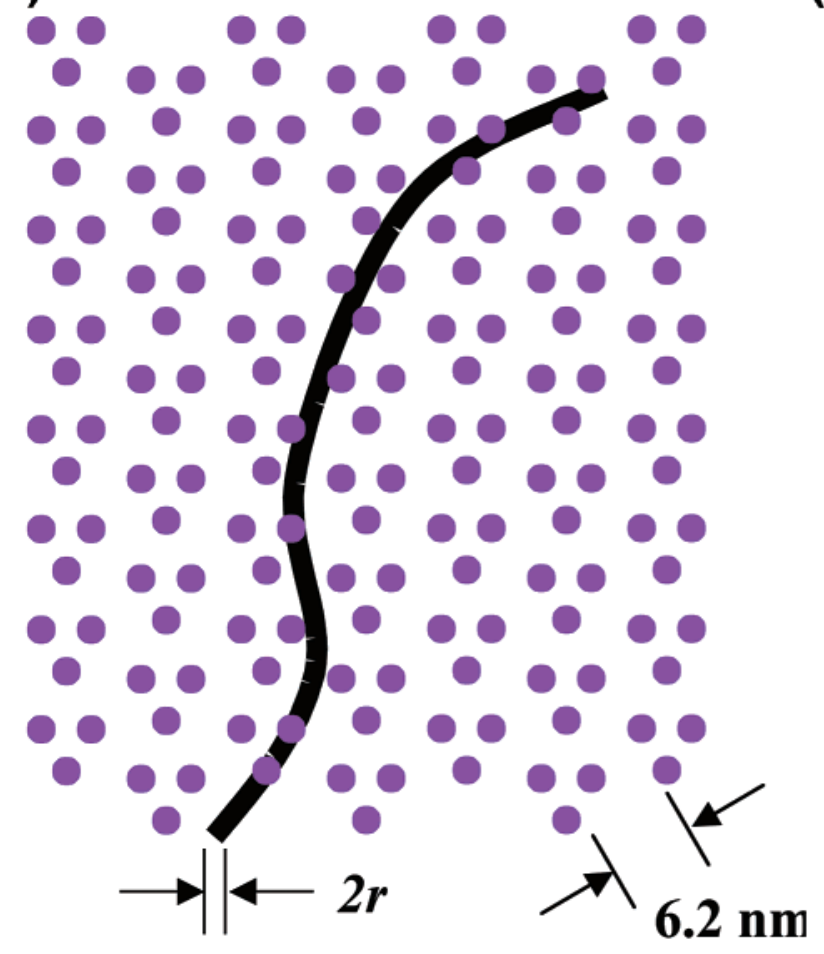

(B)

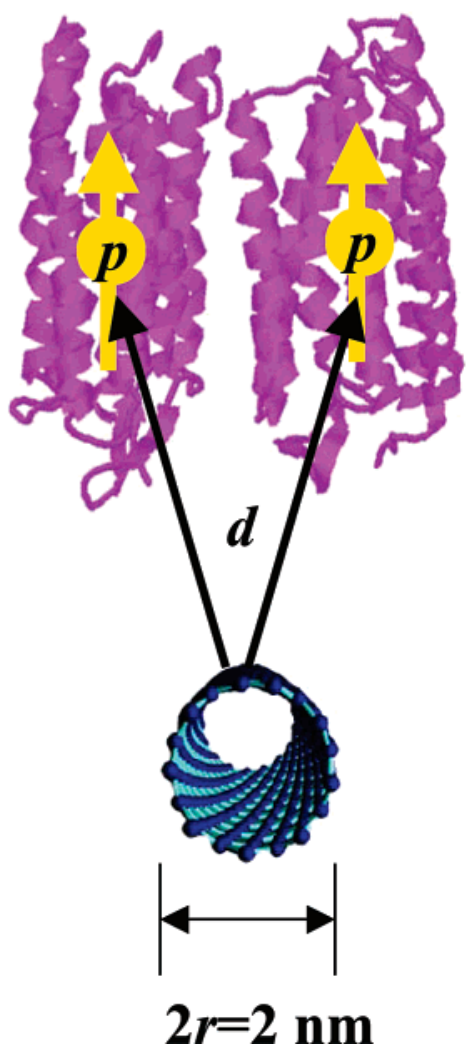

Figure 3. (A) Model illustrating the geometry of PM with respect to the nanotubes. Rhodopsin (purple dots) assembles into trimers, which are arranged on a hexagonal lattice. Each nanotube is a curved line that meanders across the lattice, contacting rhodopsins over its width of $2 \mathrm{~nm}$. Since the rhodopsin dipole density is $6.0 \times 10^{-29} \mathrm{C} \cdot \mathrm{m} / \mathrm{nm}^{2},{ }^{11}$ the nanotube contacts a line density of $\pi=1.2 \times 10^{-25} \mathrm{Cm} / \mu \mathrm{m}$ of nanotube length. (B) Model illustrating the dimensions used in the calculations. A typical nanotube has a typical diameter of 2 nm. A rhodopsin monomer situated near a nanotube has a dipole moment $p$. Although this dipole arises from a complex extended charge distribution, it is represented by a point dipole for simplicity. This point dipole is situated within the rhodopsin at a distance $d$ from the nanotube surface.

dipole on the nanotubes. ${ }^{25}$ The dipole is still not well understood, but it is known to result from the competition between several charge distributions ${ }^{6}$ that result in a net dipole moment of $3.3 \times 10^{-28} \mathrm{C} \cdot \mathrm{m}$ per rhodopsin monomer. ${ }^{11}$ To calculate the effect of this dipole on the nanotubes, we use a simple electrostatic model in which the rhodopsin molecules above a nanotube (Figure 3A) form a line of constant dipole density (see Supporting Information). In our model, the line of dipoles with a density $\pi$ induces a charge density, $\lambda$, given by $\lambda=-r \pi / d^{2}$ (Figure 3B). Thus, by combining the known dipole moment of bacteriorhodopsin with the induced charge (measured from the threshold voltage shift and the known capacitance), we calculate how far the dipoles lie from the nanotubes. The answer will be different for the two different orientations, reflecting the position of the dipoles closer to one side of the PM. For the cytoplasmic orientation, with $\Delta V_{\mathrm{cp}}=+2.2 \mathrm{~V}$, we calculate $d_{\mathrm{cp}}=1.9$ $\mathrm{nm}$. For the extracellular orientation, with $\Delta V_{\mathrm{ec}}=-0.4 \mathrm{~V}$, we have $d_{\mathrm{ec}}=4.4 \mathrm{~nm}$. Since the sum of these distances, 6.3 $\mathrm{nm}$, is comparable to the membrane bilayer thickness of 5 $\mathrm{nm}$ (ref 9), we conclude that this simple model is reasonable. Note, in particular, that since the ratio between $\Delta V_{\text {cp }}$ and $\Delta V_{\mathrm{ec}}$ is 5.5 , the electrostatic model indicates that $d_{\mathrm{cp}}$ is 2.3 times smaller than $d_{\mathrm{ec}}$. Thus, our data contribute additional details about the asymmetry of the bacteriorhodopsin charge distribution.
In conclusion, enough is now known about nanoelectronics and carbon nanotube transistors that we are able to use a nanodevice as an investigative tool. We have used the interaction between a biological system and a nanodevice not to learn about the electronic component, but to learn about the biological component. This represents a significant step in nanotechnology. In addition, the particular nanodevice we have used includes a network of nanotubes. This type of nanodevice is ideal for nanobioelectronics, in that stable nanostructured semiconductors are made available at the scale necessary to interface with biology. The device geometry, with the conducting channel of the transistor in direct contact with the biological system, allows excellent communication between the two components of the complex device. Such direct communication is not possible with conventional transistors where the conducting channel is buried within the structure. In addition, single molecule sensitivity, demonstrated with our devices ${ }^{27}$ allows improved monitoring of the interaction.

We have demonstrated that the nanoelectronic devices continue to function as transistors, that the cell membranes preserve their properties, and that these two component systems interact. As a result, it should be possible to connect living cells directly to these nanoelectronic devices. The devices also operate in a buffer environment, allowing monitoring of certain cell functions by subjecting the cell to 
different environments. Current technology allows the device size to be reduced to 20 nanometers, significantly smaller than the size of a typical cell area if immobilized onto a surface. Thus, an array of devices would allow the detailed monitoring of a living cell in a buffer. In addition, the electronic devices allow the application of local electric fields, which will likely influence cell functions, opening the way to what could be called "cellectronics", truly functional integration of electronics and biological matter.

Acknowledgment. The authors gratefully acknowledge Wayne Hubbell for providing PM suspension. We thank the Nanomix technical staff for device fabrication.

Supporting Information Available: Materials and methods and a description of the electrostatic model used. This material is available free of charge via the Internet at http:// pubs.acs.org.

\section{References}

(1) Willner, I. Science 2002, 298, 2407.

(2) Hu, H.; Ni, Y.; Montana, V.; Haddon, R. C.; Parpura, V. Nano Lett. 2004, 4, 507.

(3) Williams, K. A.; Veenhuizen, P. T. M.; de la Torre, B. G.; Eritja, R.; Dekker, C. Nature 2002, 420, 761

(4) Pantarotto, D.; Partidos, C. D.; Graff, R.; Hoebeke, J.; Briand, J.-P.; Prato, M.; Bianco, A. J. Am. Chem. Soc. 2003, 125, 6160.

(5) Zheng, M.; Jagota, A.; Strano, M. S.; Santos, A. P.; Barone, P.; Chou, S. G.; Diner, B. A.; Dresselhaus, M. S.; Mclean, R. S.; Onoa, G. B.; Samsonidze, G. G.; Semke, E. D.; Usrey, M.; Walls, D. J. Science 2003, 302, 1545. Chen, R. J.; Bangsaruntip, S.; Drouvalakis, K. A.; Kam, N. W. S.; Shim, M.; Li, Y.; Kim, W.; Utz, P. J.; Dai, H. P. N. A. S. 2003, 100, 4984 .

(6) Wang, G.; Porschke, D. J. Phys. Chem. B 2003, 107, 4632.

(7) Richard, C.; Balavoine, F.; Schultz, P.; Ebbesen, T. W.; Mioskowski, C. Science 2003, 300, 775. Besteman, K.; Lee, J.-O.; Wiertz, F. G. M.; Heering, H. A.; Dekker, C. Nano Lett. 2003, 3, 727.

(8) Bradley, K.; Gabriel, J.-C. P.; Grüner, G. Nano Lett. 2003, 3, 1353.

(9) Miyasaka, T.; Koyama, K.; Itoh, I. Science 1992, 255, 342.
(10) Oesterhelt, D.; Stoeckenius, W. Proc. Natl. Acad. Sci. U.S.A. 1973, $70,2853$.

(11) Ermolina, I.; Lewis, A.; Feldman, Y. J. Phys. Chem. B 2003, 107, 14537.

(12) Steinhoff, H.-J.; Mollaaghababa, R.; Altenbach, C.; Hideg, K.; Krebs, M.; Khorana, H. G.; Hubbell, W. L. Science 1994, 266, 105.

(13) Várò, G. Acta Biol. Acad. Sci. Hung. 1981, 32, 301.

(14) Yi, S.; Safinya, C. R.; Liang, K. S.; Ruppert, A. F.; Rothschild, K. J. Nature 1993, 366, 48 .

(15) Tans, S. J.; Verschueren, A. R. M.; Dekker. C. Nature 1998, 393, 49.

(16) Durkop, T.; Getty, S. A.; Cobas, E.; Fuhrer, M. S. Nano Lett. 2004, 4, 35 .

(17) Kim, W.; Javey, A.; Vermesh, O.; Wang, Q.; Li, Y.; Dai, H. Nano Lett. 2003, 3, 193.

(18) Tans, S. J.; Dekker, C. Nature 2000, 404, 834.

(19) Freitag, M.; Radosavljevic, M.; Zhou, Y.; Johnson, A. T.; Smith, W. F. Appl. Phys. Lett. 2001, 79, 3326.

(20) Bockrath, M.; Cobden, D. H.; McEuen, P. L.; Chopra, N. G.; Zettl, A.; Thess, A.; Smalley, R. E. Science 1997, 275, 1922.

(21) Javey, A.; Kim, H.; Brink, M.; Wang, Q.; Ural, A.; Guo, J.; McIntyre, P.; McEuen, P.; Lundstrom, M.; Dai, H. Nat. Mater. 2002, 1, 241.

(22) Bradley, K.; Gabriel, J.-C. P.; Briman, M.; Star, A.; Grüner, G. Phys. Rev. Lett. 2003, 91, 218301.

(23) This assumption, that the mixed-orientation film contains equal amounts of cytoplasmic and extracellular orientations, is justified by our observations. First, we have already concluded that our deposition method produces similar coverages for both orientations. Therefore, neither orientation adsorbs preferentially compared to the other, and a random mixture should contain equal amounts of each. Second, the threshold shift observed with mixed orientation correlates with the expectation from a 50/50\% mixture. The two oriented depositions cause $+2.2 \mathrm{~V}$ and $-0.4 \mathrm{~V}$ of threshold shift. For a $50 / 50 \%$ mixture, we expect a net threshold shift of $(1 / 2)(2.2-0.4) \mathrm{V}$, or $+0.9 \mathrm{~V}$. This value agrees well with the value observed with mixed orientation, $1.0 \pm 0.2 \mathrm{~V}$. From these two observations, we conclude that the mixed-orientation film is in fact a $50 / 50 \%$ mixture.

(24) Ehrenberg, B.; Berezin, Y. Biophys. J. 1984, 45, 663.

(25) The background charge due to the phosphate heads of the lipids is 0.2 electrons per square nanometer, ${ }^{24}$ which is too weak to explain the charge induced in our devices.

(26) Koyama, K.; Yamaguchi, N.; Miyasaka, T. Science 1994, 265, 762.

(27) Bradley, K.; Gabriel, J.-C. P.; Briman, M.; Star, A.; Grüner, G. Phys. Rev. Lett. 2003, 91, 218301.

NL050157V 